Kansas State University Libraries

New Prairie Press

\title{
THE EFFECT OF DESIGN AND DOSE LEVEL CHOICE ON ESTIMATING THE OPTIMAL DOSE IN A QUANTITATIVE DOSE- RESPONSE EXPERIMENT
}

\author{
Henry R. Rolka \\ George A. Milliken \\ James R. Schwenke \\ Marta Remmenga
}

See next page for additional authors

Follow this and additional works at: https://newprairiepress.org/agstatconference

Part of the Agriculture Commons, and the Applied Statistics Commons

\section{(c) (†) $\ominus$}

This work is licensed under a Creative Commons Attribution-Noncommercial-No Derivative Works 4.0 License.

\section{Recommended Citation}

Rolka, Henry R.; Milliken, George A.; Schwenke, James R.; and Remmenga, Marta (1991). "THE EFFECT OF DESIGN AND DOSE LEVEL CHOICE ON ESTIMATING THE OPTIMAL DOSE IN A QUANTITATIVE DOSERESPONSE EXPERIMENT," Conference on Applied Statistics in Agriculture. https://doi.org/10.4148/ 2475-7772.1425

This is brought to you for free and open access by the Conferences at New Prairie Press. It has been accepted for inclusion in Conference on Applied Statistics in Agriculture by an authorized administrator of New Prairie Press. For more information, please contact cads@k-state.edu. 
Author Information

Henry R. Rolka, George A. Milliken, James R. Schwenke, and Marta Remmenga

This is available at New Prairie Press: https://newprairiepress.org/agstatconference/1991/proceedings/13 


\author{
Henry R. Rolka \\ George A. Milliken \\ James R. Schwenke \\ Marta Remmenga \\ Kansas State University \\ Department of Statistics \\ Manhattan, Kansas 66506
}

\begin{abstract}
D-optimality is a commonly used criterion to evaluate a design with respect to parameter estimation. The variance of the optimal dose estimate is another criterion for evaluating a design. The quantitative dose-response experiment involves fitting a model to data and estimating an optimal dose. Two techniques for estimating an optimal dose and three models are used to compare the variances of optimal dose estimates over nine equally spaced balanced designs and five fixed unequally spaced six-point designs. The results show that a design which is more Doptimal than another design does not necessarily produce optimal dose estimates with less variance.
\end{abstract}

Keywords: Dose-response, optimal design, optimal dose.

\title{
1. INTRODUCTION
}

The dose-finding trial is a critical element in the successful development of most new pharmaceutical products. The importance of the correct determination of the most useful dose or dosage range for a new drug is well documented (Turri, 1986). Several methodologies are commonly used, depending on the nature of the product under evaluation. The product focus under consideration for the present study is livestock growth promotion substance.

In the process of establishing a dose at which to market a new growth promotion drug, there are economic and safety factors to consider. The company manufacturing and selling the product has the incentive to encourage use at a dose which will enhance the competitive marketability of the product. The Federal Food and Drug Administration has explicit regulatory responsibility for approving the recommended dose. The FDA is expected by the public to carefully consider safety and to discourage excess introduction of manufactured substances into the environment. In the process of striking a balance to these inherently conflicting incentives, the language and techniques of dose finding evolve.

Ideally, what is sought is an "optimal dose" such that any increase in the amount produces no detectable improvement in the growth rate and any decrease produces a dramatic 
drop in the growth rate. More realistically, an "optimal range" of doses would be considered such that increasing the dose above the range provides no significant increase in growth rate and dosing animals below the range resuits in a significant decrease in the growth rate. In this study, the term "optimal dose" will be taken to mean any dose within an "optimal range" as described. The term "dose-finding" will refer to any systematic procedure for identifying optimal doses. A more extensive discussion of the various "optimal dose" terms used can be found in Remmenga (1991), Turri (1986) and Preclearance Guidelines for Production Drugs (1975). In most instances, the characteristics of the product (ie. growth promoter, therapeutic agent, toxic vs otherwise inert, etc.) determines the nature of the optimal dose and the subsequent dose-finding methodology. A review of these methodologies can be found in Remmenga (1991).

A concern common to any dose-finding procedure based on data from a controlled study is how to decide what levels of dose to evaluate. It is first necessary to obtain a range in which the drug is known to be effective near the higher doses and not effective at the low end of the range. It is recommended by FDA guidelines that this information be obtained during Phase I dose ranging studies (Freston 1986). For the present inquiry it is assumed that the effective range of the product is over dosages from zero to three. Therefore, the estimated optimal dose is expected to be between 1.5 and 3. It has also been suggested that a zero dose be included with three to six non-zero levels (Preclearance Guidelines 1975), (Robson 1973). The FDA guidelines recommend either equal spacing between zero and the highest dose or equal spacing between the lowest non-zero dose and the highest dose. It is acknowledged, however, that for some situations "conferences should be held to determine the 'best' non-zero levels." This can be taken to mean that in some situations the response distribution over the dosage range is not totally unknown. Conditions simulated in this study include a zero dose and three equally spaced nonzero doses, as well as a zero dose with five non-zero doses which are not all equally spaced. It is assumed that the form of the underlying response distribution is known (or is well approximated by a model) to the investigators. What is sought is a set of values for the parameters which best accommodates the data and a subsequent target dose. Since in many of the dose-finding techniques the optimal dose is dependent on the model fitting process as well as the data, the number of observations at the various dose levels becomes a primary consideration in designing a dose-finding experiment. The general goal of the current study is to provide some insight into the optimal allocation of the observations in the dose-response experiment.

\section{BACKGROUND}

This study is part of a more comprehensive research endeavor to characterize and compare properties of several techniques, models and experimental designs used in dose-finding. The Bureau of Veterinary Medicine has established previously referred to guidelines for conducting experiments to determine an optimal dose at which to market a new drug. Among other things, these guidelines suggest techniques, models and spacing of doses to be used in trials. A report on the inquiry into the various techniques and four of the typical models (one linear in the parameters and three nonlinear, including spline models) used in dose response studies can be found in Remmenga (1991). In that report it was concluded: (i) that the range of response does not affect the performance of the estimation technique, (ii) that increasing the population standard deviation causes the techniques to produce lower maximum effective dose estimates, (iii) that although there are some difficulties associated with the Mitcherlich model (described later), it very likely represents many true dose-response relationships, and (iv) that the model selected 
to fit the data has a formidable influence on the target dose estimates. Consequently, the importance of careful model selection is suggested.

In an attempt to more precisely estimate the parameters of a given nonlinear model, the concept of "optimal design" enters the picture. The term "design" is defined to be any specified allocation of treatments to experimental units (Hedayat 1988). In the dose-response experimental setting, the treatments are dose levels and the experimental units are usually the individual animals or groups of animals. "Optimal" will be taken to mean "most efficient" or simply "best" with respect to estimating parameters in the model. It is important to keep in mind that although optimal dose estimation is dependent upon the estimated model parameters, a design which is optimal for estimating the model parameters may not be so for estimating the optimal dose. This idea is central to this report.

For any modeling situation, it is desirable to incorporate a design which minimizes the variance about the parameter estimates. D-optimality is a criterion for evaluating a design with respect to parameter estimates. Consider a model with additive error structure:

$$
y_{i}=f_{i}\left(\underline{x}_{i}, \underline{B}\right)+\epsilon_{i} \quad \mathrm{i}=1, \ldots, n \text {. }
$$

where $y_{i}$ is the ith response,

$\underline{x}_{i}^{\prime}=\left[x_{i 1}, x_{i 2}, \ldots, x_{i q}\right]$ is a vector of known constants,

$\underline{\beta}=\left[\beta_{1}, \beta_{2}, \ldots, \beta_{\mathrm{p}}\right]$ is a vector of $\mathrm{p}$ unknown parameters

and $\underline{E}$ is distributed with mean $\underline{0}$ and variance $\sigma^{2} \underline{I}$.

Let the matrix $\underline{D}$ have elements $d_{i j}=\frac{\partial f\left(x_{i}, \underline{\beta}\right)}{\partial \beta_{j}} i=1, \ldots n ; j=1, \ldots p$. Note that for the linear model, $\underline{D}$ is a matrix of constants where for a model nonlinear in the parameters, $\underline{D}$ depends on $\underline{\beta}$. The design which minimizes $\sigma^{2}\left|\left(\underline{D}^{\prime} \underline{D}\right)^{-1}\right|$ (equivalent to maximizing $\sigma^{2}\left|\left(D^{\prime} D\right)\right|$ is said to be D-optimal. Further, a p-parameter model has a D-optimal design with p design points.

Extensive comparisons of optimality criteria can be found in Federov (1972), Pazman (1986), and St. John and Draper (1975). Unfortunately, an approach to developing an experimental design which simultaneously satisfies all optimality criteria doesn't appear to exist. Since the D-optimality criterion compares the results of an experiment over different spacings of the independent variable (given the model), it is an appropriate evaluative consideration for determining the optimal design in a dose-finding experiment when employing a regression based approach. A design is D-optimal if and only if it is G-optimal (St. John and Draper, 1975), where G-optimality ensures that the particular design minimizes the maximum variance for any predicted value over the range of doses in the design. It has also been documented that designs which are D-optimal are nearly optimal with respect to many other criteria (Dodge, Federov, Wynn 1988). Hill (1980) showed that when a model is linear in some of its parameters, the Doptimal design is independent of the values of those parameters.

An application of the D-optimality criterion for selecting a design to evaluate the grade of beef by modeling post-slaughter $\mathrm{pH}$ decline is described in Kendall (1990). The nonlinear decay model and initial parameter ranges are assumed to be known. An algorithm is successfully employed to select design points so as to minimize the variance about the parameter estimates using the D-optimality criterion. The procedure seems to work for parameter estimation and 
maximizing local power for the F-ratio in tests about the parameters (Wald 1943), but how useful is this parameter estimation design criterion in relation to optimal dose estimation? In an attempt to answer this question, different models and techniques for estimating optimal doses were examined over a variety of designs with respect to the variance about optimal dose estimations.

\section{METHODOLOGY}

\subsection{MODELS}

Three models were utilized in this study. They are:

(i) Quadratic model: $y=\beta_{0}+\beta_{1} X+\beta_{2} X^{2}+\epsilon$

(ii) Mitcherlich model: $y=\beta_{0}-\beta_{0} \beta_{1} \mathrm{e}^{-\beta_{2} x}+\epsilon$

(iii) Linear-Linear Plateau model:

$$
y= \begin{cases}\beta_{0}+\beta 1 X+\epsilon, & \text { if } X<\beta_{2} \\ \beta_{0}+\beta_{1} \beta_{2}+\epsilon, & \text { if } X \geq \beta_{2}\end{cases}
$$

These functional forms were selected because they are commonly used for representing the quantitative dose response relationship. Parameters for generated response levels were selected to represent the stage of a drug evaluation study where the range of doses within which the dose that elicits the maximum is located has already been determined (or for the Mitcherlich model, the response has at least leveled off near the upper end of the dosage range). Without loss of generality, a dosage range of $\mathrm{X}=0$ to 3 was used and the parameter values selected for the simulation portion of the study were based on having responses range from 10 to 20 .

\subsection{TECHNIQUES FOR OPTIMAL DOSE ESTIMATION}

In order to assess the effects of design choice on optimal dose estimation, it was necessary to select techniques for dose finding. Selection of the techniques was based on results of a previous study which compared a variety of such techniques across different models, variances and response levels (Remmenga 1991). Two of the techniques that were found to perform reasonably well in most cases are the Test for Slope Equal to Zero technique (TSZ) and the Lower Limit of the Maximum Response technique (LLM). These two techniques were used in this study. 
The TSZ approach is based on considering an optimal dose to be the dose above which no further improvement in growth is obtained. The estimated optimal dose obtained by this technique is called $X$ Where the Slope Equals Zero (XSEZ). The XSEZ is found by fitting a model to the data and establishing which dose is such that any further increase in the dose causes the slope of the response curve to no longer be significantly different from zero at the $\alpha=.05$ level. The TSZ technique is especially useful when a model such as the Mitcherlich model is fit which has an asymptotic maximum. Note that in the Linear-Linear Plateau model, the join-point is comparable to XSEZ.

The LLM technique results in the lowest dose which may elicit the maximum response of the fitted model. The resulting optimal dose estimate is referred to here as the $\mathrm{X}$ Which Elicits the Lower Limit of the Maximum Response (XLMAX). The XLMAX is found by first fitting the data to a model and then constructing a confidence interval about the maximum of the fitted model. Then the inverse of the response function is solved for the dose which elicits the lower limit of the confidence interval about the maximum response. That dose is the XLMAX.

\subsection{DESIGNS}

In order to reduce the number of factors to be considered, an assumption was made to have a total sample size of $N=60$. The number 60 can be factored in a variety of ways which enabled comparison of many equally spaced designs. Two general approaches for allocating observations were employed. One approach (Method $A$ ) divides the design space into increasing numbers of segments with decreasing numbers of observations at each design point as shown in Table 1.

The other method (Method B) considers a fixed number of design points at fixed doses and varies the numbers of observations at each of the fixed design points as shown in Table 2 . For example, D13 4 is a fixed six-point design which has $n=13$ observations at the control dose, and doses of 1,2 and 3 , with $n=4$ observations allocated at doses of 0.5 and 1.5 .

\section{PROCEDURES}

Interest was in exploring design selection for allocating 60 observations in a way that maximizes model estimation accuracy, to include testing for lack of fit, as well as precisely identifying an optimal dose. D-optimality criteria and the variances about typical optimal dose estimators were obtained for the designs produced by Methods A and B and the models shown in section 3.1. Without loss of generality, $\sigma^{2}$ was assumed to be one for calculating variances about estimators and D-optimality criteria.

Since the dose which theoretically elicits the maximum in the Mitcherlich model is at infinity, the variance about the slope of the response function was used for comparison at am arbitrary dose $(x=2)$ toward the upper end of the dosage range $(0-3)$, instead of using the variance about a maximum response. The variance about the join-point was used to compare the Linear-Linear Plateau model. For the Quadratic model, the dose that elicits the maximum response was obtained directly by setting the first derivative to zero and solving for $X$. The variance about the resulting $X_{0}$ was then calculated for each design. For the designs in Table 1 , 
three different maximum doses and join-points were used in the Quadratic and Linear-Linear Plateau models, respectively.

The programs were written in GAUSS (Aptech Systems, Inc.) version 2.0 programming language and were run on a Compaq Deskpro 386/20e personal computer. Copies of these programs can be found in Rolka (1991).

\section{FINDINGS}

Since the point at which the "slope equals zero" is comparable in the Mitcherlich model to the join-point of the Linear-Linear Plateau model, the variance about the slope at a particular dose in the Mitcherlich was compared to the variance about the join-point over the fixed six-point designs of Method $B$. The data were generated by calculating linearized approximations to the variance of the slope using $\beta_{0}=20, \beta_{1}=0.5$ and $\beta_{2}=1.28$ in model (ii). Variances for the slope and the D-optimality criteria for the Mitcherlich model are shown in Table 3 for the designs produced by Method B.

The Det $\left(\underline{D}^{\prime} \underline{D}\right)^{-1}$ gets larger as the number of observations at the intermediate doses (ie. $X=0.5$ and $X=1.5$ ) increases. If one were to use the D-optimality criterion to select a design from the six evaluated, either D15_0, D14_2 or D13_4 would be the most likely choices because the determinant is the same out to 8 decimal places for those three designs and is smaller than the determinant for the other 3 designs. But when considering the minimum variance about the slope of the curve as an evaluative criterion, D11_8 performs best. This can be seen clearly in Figure 1 .

Table 4 shows the D-optimality criterion and variance of the join-point for the LinearLinear Plateau model across the designs of Method $B$. The parameters used for the parameterization shown in model (iii) were $\beta_{0}=10, \beta_{1}=6.6667$ and $\beta_{2}=$ Join-Point $=1.5$. The variance of the join-point was calculated by selecting out the element of $\left(\underline{D} \underline{D}^{-1}\right.$ which corresponded to the parameter that represented the join-point. For this model, the D-optimality criterion decreases with an increasing number of observations at intermediate doses, as does the variance about the join-point (see Figure 2).

Take notice that one of the intermediate doses (ie. $X=1.5$ ) is located at the join-point. This raises the question of whether the variance about the join-point estimate would be influenced over designs if this were not the case.

In order to address this question, the Linear-Linear Plateau model was used with the designs produced by Method A. (Those were equally spaced and varied the distance from the dose that elicited the maximum response (JP) and the nearest design point.) Since the Mitcherlich model did not have a tangible value which elicited the maximum response in the dosage range, for the sake of comparison across designs of Method A the Quadratic model was used, since a 'maximum dose' could be easily determined for a given set of parameter values.

Table 5 shows the join points, number of design points, D-optimality criteria and variances about the join-point estimates for the 9 designs produced by method $A$. 
Recall that each of the 9 designs were equally spaced and the design points were different from design to design. Notice that for the case where the join-point parameter was 1.5 in the equally spaced 6-point design, $\operatorname{Det}\left(\underline{D}^{\prime} \underline{D}\right)^{-1}=0.000003472$ and the variance for the estimator of the join point $=0.004031250$. This design had 10 observations at each of doses $0,0.6,1.2$, 1.8, 2.4 and 3. The design with 10 observations at doses $0,0.5,1,1.5,2$ and 3 (Table 4) with the Linear-Linear model having a join-point at 1.5 had $\operatorname{Det}\left(\mathrm{D}^{\prime} \underline{\mathrm{D}}\right)^{-1}=0.00000225$ and a variance about the join-point estimator of 0.0027 . If the join-point is at 2.5 for the equally spaced 6-point design, Det $\left(\underline{D}^{\prime} \underline{D}\right)^{-1}=0.000003472$ and $\operatorname{Var}($ join-point estimator $)=0.010434028$. There were differences noted in both the D-optimality criteria and the variances of the join-point estimator. Thus the variance of the join-point estimator is not only sensitive to the particular value of the estimator at which it is evaluated, it is also dependent on how close to that value an observation happens to fall. This finding can be seen in Figure 3. The variance of the join-point is labile in the Linear-Linear Plateau model with respect to equally spaced designs when the number of design points is less than 10 .

Table 6 shows the dose which elicits the maximum response ('Max-Dose'), the number of design points, Det $\left(\underline{D}^{\prime} \underline{D}\right)^{-1}$ and Var('Max-Dose' estimator) for Quadratic models with 3 different maximum response locations for the 9 designs of method A. Figure 4 shows that for the Quadratic model the variance of the estimated dose that elicited the maximum response (ie. 'Max-Dose') depended on the value at which that estimator was evaluated, however, there was much more stability with respect to the designs.

In order to substantiate the patterns of variance changes over designs produced by Method $B$ (shown in Figures 1 and 2), a simulation was conducted to represent a series of estimation events using the fixed 6-point designs with the Mitcherlich and Linear-Linear Plateau models.

\section{SIMULATION RESULTS}

Mitcherlich data was generated with $\beta_{0}=20, \beta_{1}=0.5$ and $\beta_{2}=1.28$ and random error added (at 3 variance levels which were increments of the control mean response: $\sigma=1.5,2$ and 2.5 ) and fit with a Mitcherlich model by a least squares iterative technique. Optimal doses were then estimated by the TSZ and LLM techniques. Similarly, the process was applied to the LinearLinear Plateau model with $\beta_{0}=10, \beta_{1}=\frac{50}{9}$ and $\beta_{2}=$ join-point $=1.8$. One thousand cases were generated for each model/design/variance combination. The cases for which a lack of fit test for the estimated model was significant at the .05 level were deleted. If the optimal dose estimates were greater than 4 , missing values were assigned. This was done based on the assumption that the effective range of the drug had already been identified and if the optimal dose was so far above the identified range, the dose ranging study should be repeated using larger doses. If the optimal dose estimates were between 3 and 4 , they were set to 3 (since the response data was generated over the range of dose values 0 to 3 and FDA guidelines would not approve a dose above the range of tested doses). The resulting sample sizes are shown in Table 7 . The data generating programs, model fitting schemes with the optimal dose estimation programs can be found in Rolka (1991). The programs were adapted from a precursor to this study reported on by Remmenga (1991). She includes a comprehensive evaluation of the performance of the original programs in that paper. Figures $5,6,7$, and 8 show the patterns of the optimal dose estimation variances over the 6-point designs of table 2, for the 2 models and 2 optimal dose estimation techniques. The patterns of the variances generally support what was shown earlier; that are summarized in figures 1 and 2 . There is less variance about the optimal dose estimates 
for the Linear-Linear model than for the Mitcherlich model. It appears that variances about estimates are generally lower as the number of observations at the intermediate doses increases. The exceptions are for going from 8 to 10 observations at intermediate doses with the intermediate variance increment and the Linear-Linear model. There is a slight increase noted. Also, for the higher variance increment, D12_6 does worse than D13_4 in each case.

\section{CONCLUSIONS}

A design that is more $\mathrm{D}$-optimal than another design is not necessarily more efficient or better for estimating the optimal dose in a quantitative dose-response study. It was shown that if $N=60$, the Mitcherlich model with the given set of parameters can adequately describe the data and an optimal dose is being estimated by the TSZ technique, the 6-point design with 8 observations at 'intermediate' doses should be considered to minimize the variance about the slope. If the Linear-Linear Plateau model could be considered reasonable to represent some true state of nature, selection of a 'good' design for estimating the join-point depends on knowing where the join-point is located! The Quadratic model showed a stable pattern of variance about the dose that elicits the maximum response, over designs which increasingly departed from the most D-optimal 3-point design.

In summary, the true nature of the dose-response relationship, the form of the model under consideration and the region of the parameters of the selected model (if nonlinear) are factors in selecting a design to estimate an optimal dose. Increments of information that become available on any of these factors in the process of dose ranging studies should be used to reevaluate the choice of design for the optimal dose estimation trial.

*This project was partially supported by the Upjohn Company and the Animal Health Institute. 


\section{WORKS CITED}

Dodge Y. Federov V., Wynn H., Optimal Design and Analysis of Experiments, Elsevier Science Pub. Co., 1988.

Federov V., Theory of Optimal Experiments, Academic Press, N.Y. 1972.

Freston J., "Dose-Ranging in Clinical Trials: Rationale and Proposed Use with Placebo or Positive Controls," The American Journal at Gastroenterology, Vol. 81, No. 5, 1986.

Hedayat A., et al, "Optimal Designs for Comparing Test Treatments with Controls," Statistical Science, Vol. 3, No. 4, 1988.

Hill P., "D-Optimal Designs for Partially Nonlinear Regression Models," Technometrics, Vol. 22, No. 2, 1980.

Kendall J., Personal Communication, 1990.

Pázman A., Foundations of Optimum Experimental Design, Kluwer Academic Publishers, MA, 1986.

"Preclearance Guidelines for Production Drugs," Bureau of Veterinary Medicine, Food and Drug Admin., 1975.

Remmenga M., "Estimating the Optimal Dose in a Quantitative Dose-Response Experiment," Master's Report, Kansas State University, 1991.

Robson D., "A Manual of Statistical Methods for Determining Dose at a Leveling-Off Point in the Growth Response to Experimental Concentrations of Antibiotic Feed Additives, "Cornell University, Biometric Unit, 1973.

Rolka, H., "The Effect of Design and Dose Level Choice on Estimating the Optimal Dose in a Quantitative Dose-Response Experiment," Master's Report, Kansas State University, 1991.

St. John R., Draper N., "D-Optimality for Regression Designs: A Review," Technometrics, Vol. 17, No. 1, 1975.

Turri M., Stein G., "The Determination of Practically Useful Doses of New Drugs: Some Methodological Considerations," Statistics in Medicine, Vol. 5, 1986.

Wald A., "On the Efficient Design of Statistical Investigations," Annals of Mathematical Statistics, Vol. 14, 1943. 
Table 1. Designs Produced by Method A

Number of Design Points

\begin{tabular}{c|c|c|c|c|c|c|c|c}
\hline 3 & 4 & 5 & 6 & 10 & 15 & 20 & 30 & 60 \\
\hline 20 & 15 & 12 & 10 & 6 & 4 & 3 & 2 & 1 \\
\hline
\end{tabular}

Number of Observations

Table 2 Designs Produced by Method B

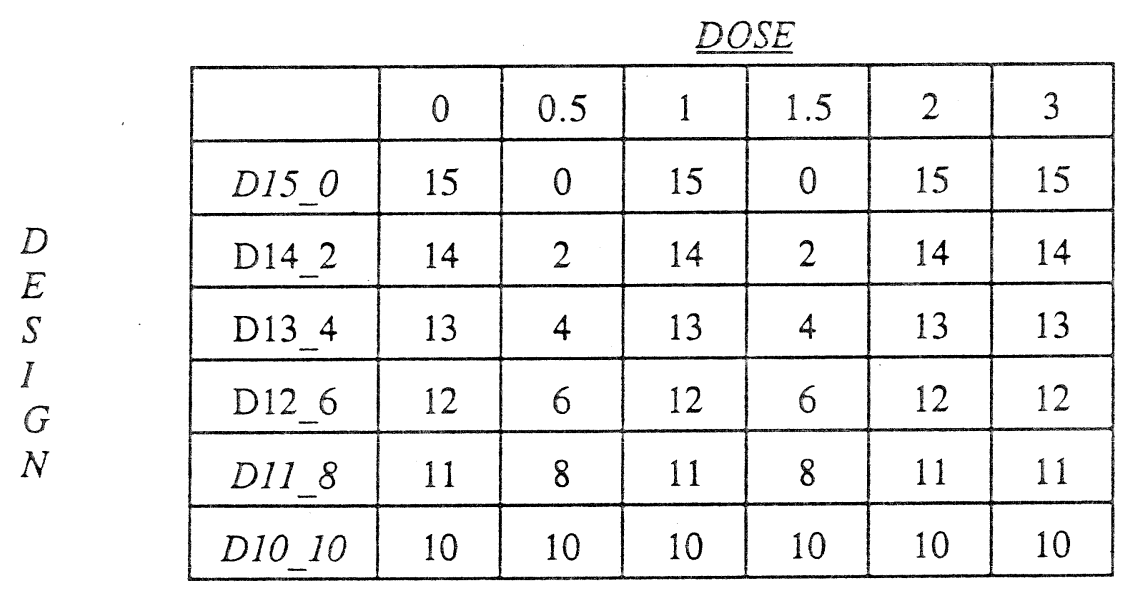

Number of Observations

Table 3. Optimality Criteria for Mitcherlich Model over Designs Produced by Method B

\begin{tabular}{c|c|c}
\hline DESIGN & DET( $\left.\underline{D}^{\prime} D\right)^{-1}$ & $\widehat{V A R(\text { Slope })}$ \\
\hline D15_0 & 0.00000009 & 0.03719762 \\
\hline D14_2 & 0.00000009 & 0.03523011 \\
\hline D13_4 & 0.00000009 & 0.03389168 \\
\hline D12_6 & 0.00000010 & 0.03306200 \\
\hline D11_8 & 0.00000010 & 0.03160224 \\
\hline D10_10 & 0.00000011 & 0.03270008 \\
\hline
\end{tabular}


Table 4 Optimality Criteria for Linear-Linear Plateau Model over Designs Produced by Method B

\begin{tabular}{|c|c|c|}
\hline DESIGN & $D E T\left(\underline{D}^{\prime} D\right)^{-1}$ & $V A R\left(\widehat{J o i n}_{\text {-Point }}\right)$ \\
\hline D15_0 & 0.00000333 & 0.00450000 \\
\hline D14_2 & 0.00000283 & 0.00373491 \\
\hline D13_4 & 0.000000254 & 0.00327374 \\
\hline D12_6 & 0.00000237 & 0.00298295 \\
\hline D11_8 & 0.00000237 & 0.00290994 \\
\hline D10_10 & 0.00000225 & 0.00270000 \\
\hline
\end{tabular}

Table 5 Optimality Criteria for Linear-Linear Plateau Model over Designs Produced by Method A

\begin{tabular}{|c|c|c|c|}
\hline $\begin{array}{l}\text { JOIN } \\
\text { POINT }\end{array}$ & $\begin{array}{l}\text { DESIGN } \\
\text { POINTS }\end{array}$ & $D E T\left(\underline{D}^{\prime} D\right)^{-1}$ & $\widehat{V A R}($ Join-Point $)$ \\
\hline 1.5 & 3 & 0.000001250 & 0.002250000 \\
\hline 2.0 & 3 & 0.000002222 & 0.005777778 \\
\hline 2.5 & 3 & 0.000003472 & 0.013194444 \\
\hline 1.5 & 4 & 0.000003333 & 0.004500000 \\
\hline 2.0 & 4 & 0.000001975 & 0.004888889 \\
\hline 2.5 & 4 & 0.000003086 & 0.010243056 \\
\hline 1.5 & 5 & 0.000001929 & 0.002500000 \\
\hline 2.0 & 5 & 0.000003429 & 0.007407407 \\
\hline 2.5 & 5 & 0.000003215 & 0.010011574 \\
\hline 1.5 & 6 & 0.000003472 & 0.004031250 \\
\hline 2.0 & 6 & 0.000002778 & 0.005688889 \\
\hline 2.5 & 6 & 0.000003472 & 0.010434028 \\
\hline 1.5 & 10 & 0.000003750 & 0.003843750 \\
\hline 2.0 & 10 & 0.000002834 & 0.005317460 \\
\hline 2.5 & 10 & 0.000003875 & 0.010478671 \\
\hline
\end{tabular}




\begin{tabular}{|c|c|c|c|}
\hline $\begin{array}{l}\text { JOIN } \\
\text { POINT }\end{array}$ & $\begin{array}{l}\text { DESIGN } \\
\text { POINTS }\end{array}$ & $D E T\left(\underline{D}^{\prime} \underline{D}^{-1}\right.$ & $\widehat{V A R}\left(\widehat{J o i n}_{\text {-Point })}\right.$ \\
\hline 1.5 & 15 & 0.000003255 & 0.003147321 \\
\hline 2.0 & 15 & 0.000003300 & 0.005831650 \\
\hline 2.5 & 15 & 0.000004131 & 0.010665550 \\
\hline 1.5 & 20 & 0.000004052 & 0.003772727 \\
\hline 2.0 & 20 & 0.000003588 & 0.006186406 \\
\hline 2.5 & 20 & 0.000004267 & 0.010765591 \\
\hline 1.5 & 30 & 0.000004172 & 0.003760045 \\
\hline 2.0 & 30 & 0.000003513 & 0.005908104 \\
\hline 2.5 & 30 & 0.000004493 & 0.011058360 \\
\hline 1.5 & 60 & 0.000004302 & 0.003752503 \\
\hline 2.0 & 60 & 0.000003628 & 0.005952053 \\
\hline 2.5 & 60 & 0.000004643 & 0.011152128 \\
\hline
\end{tabular}

Table 6 Optimality Criteria for Quadratic Model over Designs Produced by Method A

\begin{tabular}{c|c|c|c} 
'MAX-DOSE' & $\begin{array}{c}\text { DESIGN } \\
\text { POINTS }\end{array}$ & DET(D'D)-1 & $\hat{\text { VAR(Slope) }}$ \\
\hline 1.5 & 3 & 0.000002743 & 0.000041088 \\
\hline 2.0 & 3 & 0.000002743 & 0.000320073 \\
\hline 2.5 & 3 & 0.000002743 & 0.002243953 \\
\hline 1.5 & 4 & 0.000003704 & 0.000049306 \\
\hline 2.0 & 4 & 0.000003704 & 0.000370370 \\
\hline 2.5 & 4 & 0.000003704 & 0.002551020 \\
\hline 1.5 & 5 & 0.000004645 & 0.000054785 \\
\hline 2.0 & 5 & 0.000004645 & 0.000415152 \\
\hline 2.5 & 5 & 0.000004645 & 0.002871960 \\
\hline 1.5 & 6 & 0.000005468 & 0.000058698 \\
\hline 2.0 & 6 & 0.000005468 & 0.000451124 \\
\hline 2.5 & 6 & 0.000005468 & 0.003142378 \\
\hline 1.5 & 10 & 0.000007748 & 0.000067236 \\
\hline
\end{tabular}




\begin{tabular}{c|c|c|c} 
'MAX-DOSE' & $\begin{array}{c}\text { DESIGN } \\
\text { POINTS }\end{array}$ & DET( $\left.\underline{D}^{\prime}\right)^{-1}$ & $\hat{V A R(\text { Slope })}$ \\
\hline 1.5 & 3 & 0.000002743 & 0.000041088 \\
\hline 2.0 & 10 & 0.000007748 & 0.000540123 \\
\hline 2.5 & 10 & 0.000007748 & 0.003841025 \\
\hline 1.5 & 15 & 0.000009314 & 0.000071905 \\
\hline 2.0 & 15 & 0.000009314 & 0.000594887 \\
\hline 2.5 & 15 & 0.000009314 & 0.004286036 \\
\hline 1.5 & 20 & 0.000010237 & 0.000074350 \\
\hline 2.0 & 20 & 0.000010237 & 0.000525356 \\
\hline 2.5 & 20 & 0.000010237 & 0.004537556 \\
\hline 1.5 & 30 & 0.000011268 & 0.000076875 \\
\hline 2.0 & 30 & 0.000011268 & 0.000658139 \\
\hline 2.5 & 30 & 0.000011268 & 0.004810954 \\
\hline 1.5 & 60 & 0.000012422 & 0.000079482 \\
\hline 2.0 & 60 & 0.000012422 & 0.000693466 \\
\hline 2.5 & 60 & 0.000012422 & 0.005108483 \\
\hline & & &
\end{tabular}

Table 7 Sample Sizes and Optimal Dose Estimation Means for Simulation

\begin{tabular}{|c|c|c|c|c|c|c|c|c|c|}
\hline \multirow[b]{2}{*}{$D E S I G N$} & \multirow[b]{2}{*}{ SIGMA } & \multicolumn{4}{|c|}{ Mitcherlich } & \multicolumn{4}{|c|}{ Linear-Linear } \\
\hline & & $n_{-} X S E Z$ & $n_{-} X L M A X$ & $\bar{X}_{-} S E Z$ & $\bar{X} \_$LMAX & n_XSEZ & $n_{-} X L M A X$ & $\bar{X} \_S E Z$ & $\bar{X} \_L M A X$ \\
\hline D10_10 & 1.5 & 719 & 954 & 2.963 & 1.963 & 966 & 966 & 1.817 & 1.711 \\
\hline D11_8 & 1.5 & 684 & 931 & 2.958 & 1.979 & 950 & 950 & 1.813 & 1.712 \\
\hline D12_6 & 1.5 & 690 & 945 & 2.948 & 2.005 & 957 & 957 & 1.816 & 1.719 \\
\hline D13_4 & 1.5 & 717 & 942 & 2.934 & 2.008 & 947 & 947 & 1.816 & 1.722 \\
\hline D14_2 & 1.5 & 727 & 953 & 2.908 & 2.025 & 962 & 962 & 1.821 & 1.730 \\
\hline D15_0 & 1.5 & 744 & 939 & 2.894 & 2.041 & 953 & 953 & 1.831 & 1.739 \\
\hline D10_10 & 2.0 & 923 & 953 & 2.734 & 1.734 & 958 & 958 & 1.827 & 1.684 \\
\hline D11_8 & 2.0 & 927 & 954 & 2.716 & 1.751 & 965 & 965 & 1.824 & 1.687 \\
\hline D12_6 & 2.0 & 926 & 955 & 2.689 & 1.760 & 967 & 967 & 1.817 & 1.685 \\
\hline D13_4 & 2.0 & 913 & 951 & 2.672 & 1.795 & 960 & 960 & 1.829 & 1.700 \\
\hline D14_2 & 2.0 & 913 & 947 & 2.648 & 1.811 & 958 & 958 & 1.837 & 1.711 \\
\hline D15_0 & 2.0 & 905 & 938 & 2.555 & 1.792 & 959 & 959 & 1.834 & 1.708 \\
\hline
\end{tabular}




\begin{tabular}{c|c|c|c|c|c|c|c|c|c|} 
& & \multicolumn{5}{|c|}{ Mitcherlich } & \multicolumn{4}{c|}{ Linear-Linear } \\
\hline DESIGN & SIGMA & $n_{\text {_XSEZ }}$ & $n_{-}$XLMAX & $\bar{X}_{-} S E Z$ & $\bar{X}_{-}$LMAX & $n_{-}$XSEZ & $n_{-}$XLMAX & $\bar{X}_{-} S E Z$ & $\bar{X}_{-}$LMAX \\
\hline D10_10 & 2.5 & 939 & 939 & 2.391 & 1.537 & 957 & 957 & 1.819 & 1.637 \\
\hline D11_8 & 2.5 & 939 & 943 & 2.397 & 1.570 & 965 & 966 & 1.834 & 1.655 \\
\hline D12_6 & 2.5 & 947 & 956 & 2.361 & 1.580 & 956 & 956 & 1.839 & 1.667 \\
\hline D13_4 & 2.5 & 944 & 948 & 2.365 & 1.604 & 958 & 958 & 1.848 & 1.681 \\
\hline D14_2 & 2.5 & 935 & 939 & 2.320 & 1.611 & 954 & 954 & 1.843 & 1.683 \\
\hline D15_0 & 2.5 & 953 & 958 & 2.276 & 1.621 & 966 & 966 & 1.858 & 1.694 \\
\hline
\end{tabular}


VARIANCE OF SLOPE PLOTTED ACROSS DESIGNS MITCHERLICH MODEL: $B O=20 B 1=0.5 B 2=1.28$ DOSE RANGE: $0-3$ / SLOPE EVALUATED AT DOSE $=2$

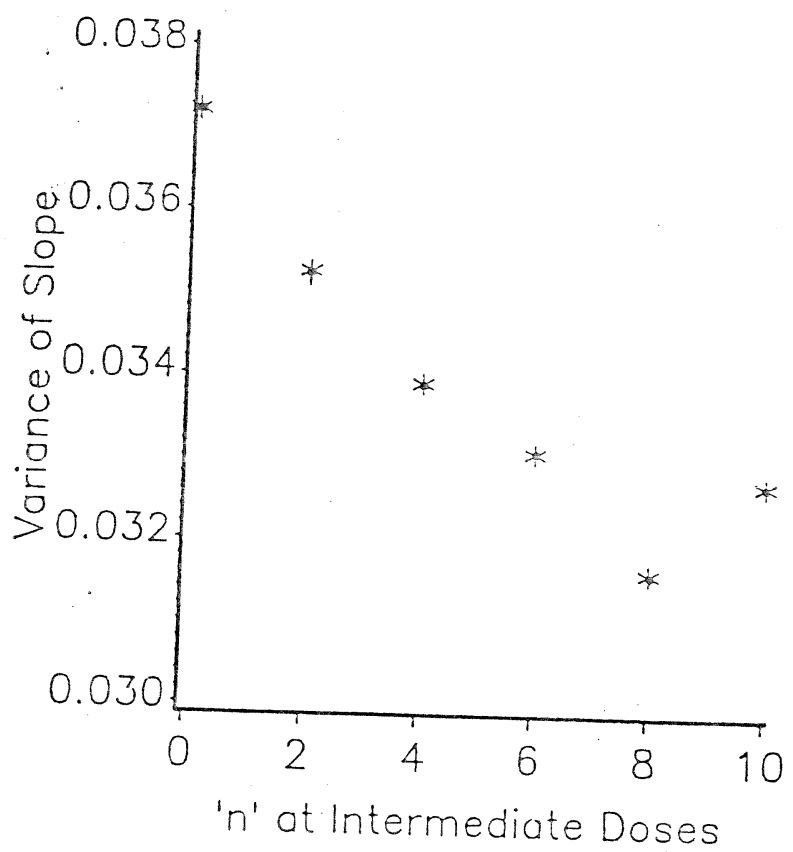

FIGURE 1

VARIANCE OF JOIN-POINT PLOTTED ACROSS DESIGNS LINEAR - LINEAR MODEL: MAX. RESPONSE $=20$ DOSE RANGE: $0-3 / \mathrm{JOIN}-\mathrm{POINT}$ DOSE $=1.5$

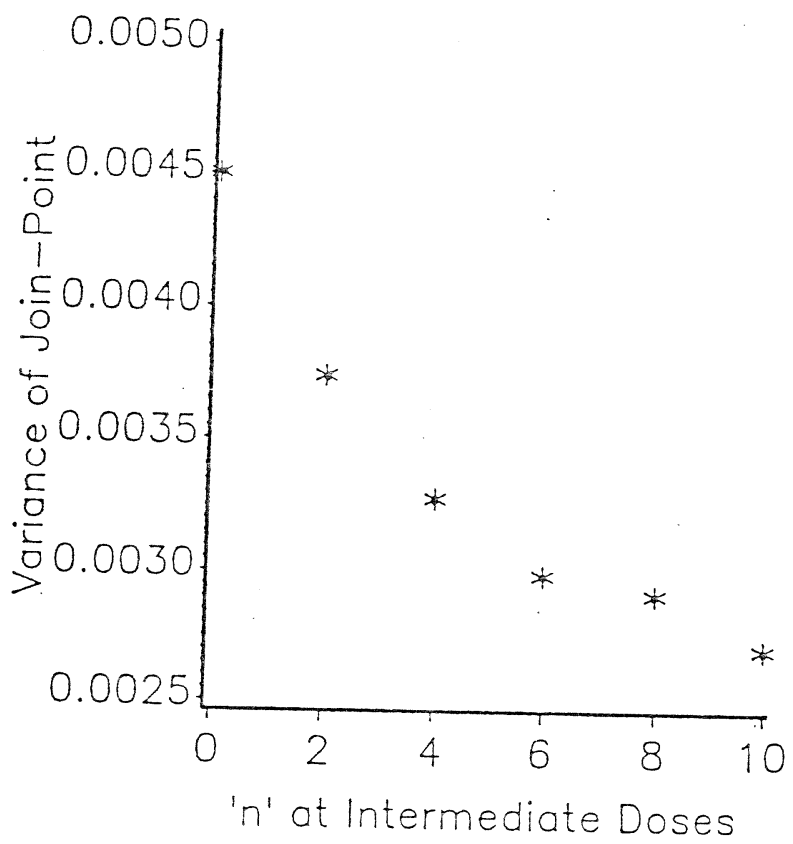

FIGURE 2 
VARIANCE OF JOIN-POINT PLOTTED ACROSS DESIGNS UNEAR-UNEAR MODEL: MAX. RESPONSE -20 DOSE RANGE: $0-3$

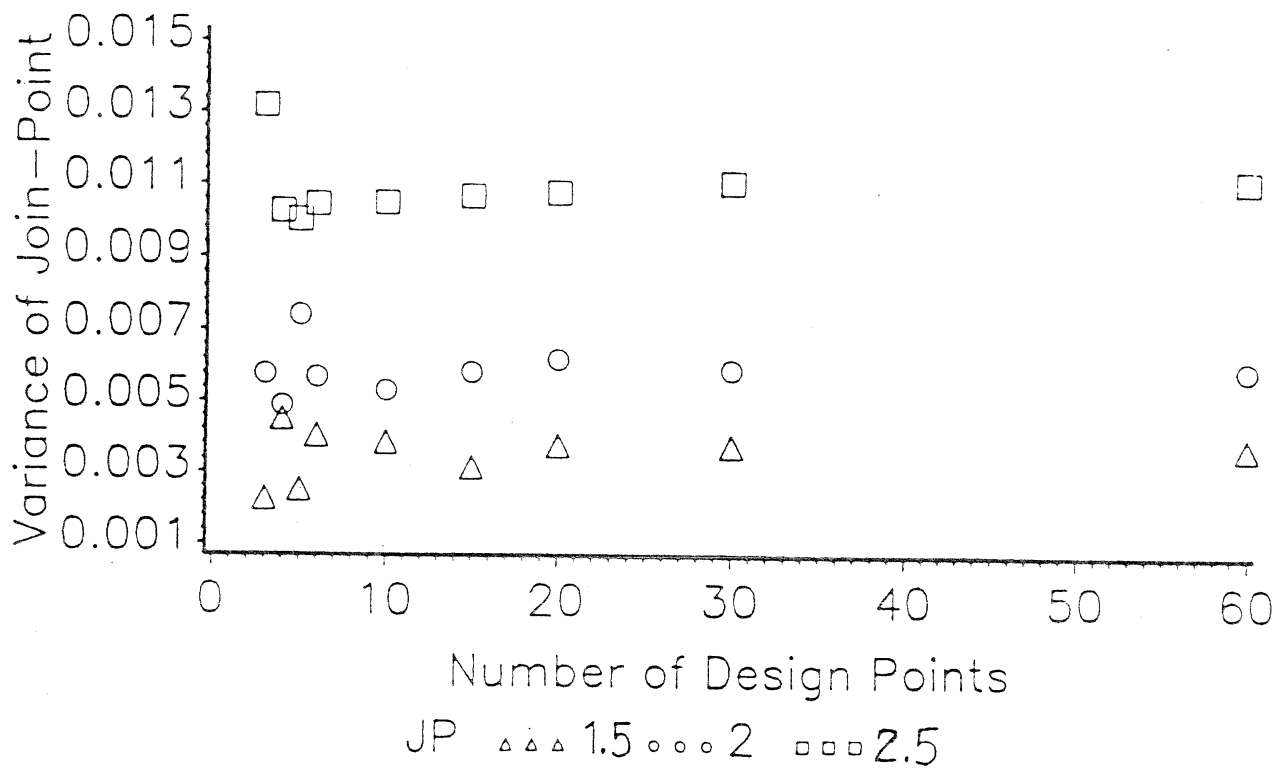

FGURE 3

VARIANCE OF 'MAXIMUM DOSE' PLOTTED ACROSS DESIGNS QUADRATIC MODEL: MAX. RESPONSE $=20$ DOSE RANGE: $0-3$

0.00550

is 0.00495

0.00440

0.00385

0.00330

0.00275

0.00220

$\geq 0.00165$

0.00110

0.00055

$\cup 0.00000$

$\frac{5}{5}$

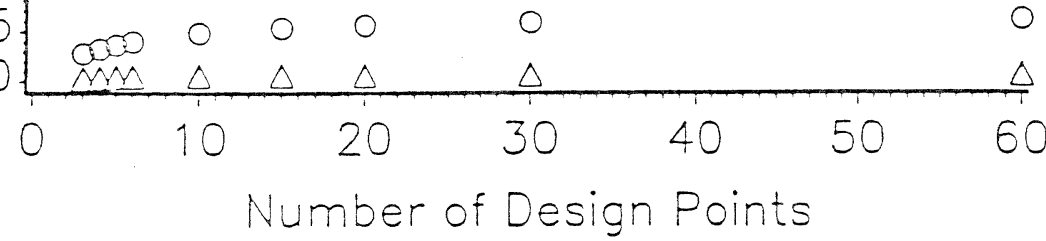

MAX_DOSE $\triangle \Delta \Delta 1.50002 \quad 002.5$

FIQURE 4 
RESULTS FROM SIMULATION: 6-POINT DESIGNS 'SLOPE $=0$ ' OPTIMAL DOSE ESTIMATION TECHNIQUE MITCHERLICH MODEL: $B O=20 \mathrm{~B} 1=0.5 \mathrm{B2}=1.28$

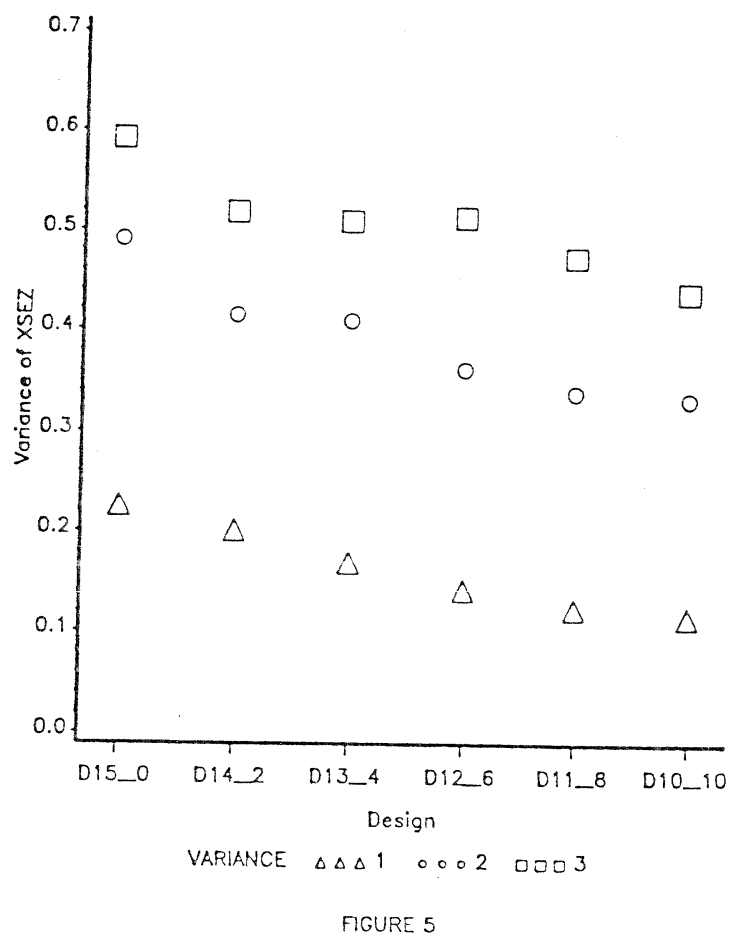

RESULTS FROM SIMULATION: 6-POINT DESIGNS 'MAX RESPONSE' OPTIMAL DOSE ESTIMATION TECHNIQUE MITCHERLICH MODEL: $B O=20$ B1 $=0.5 B 2=1.28$

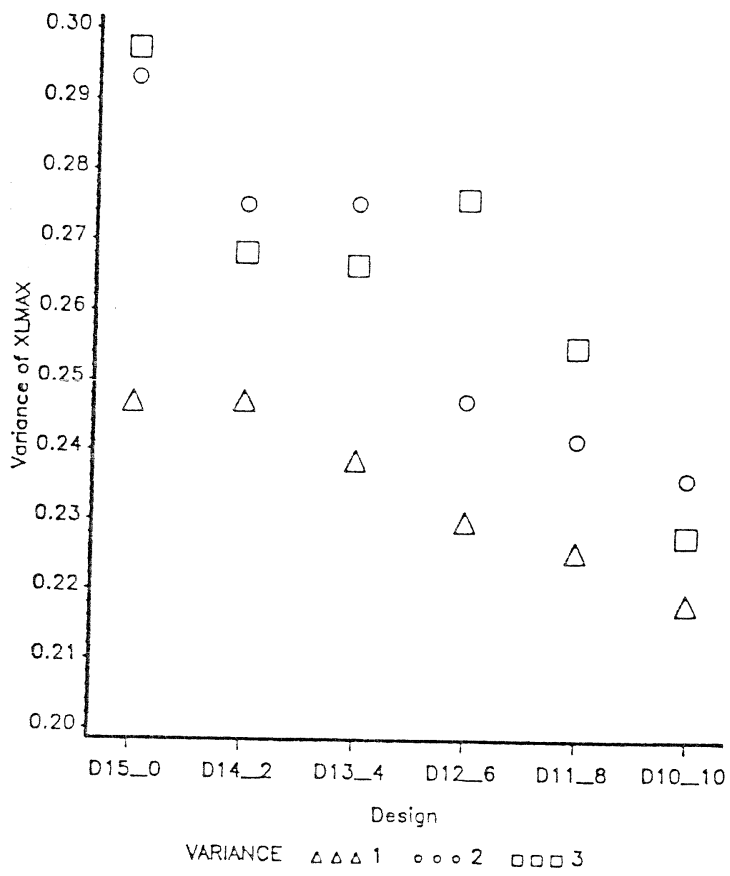

FIGURE 6 
RESULTS FROM SIMULATION: 6-POINT DESIGNS 'SLOPE $=$ O' OPTIMAL DOSE ESTIMATION TECHNIQUE

LINEAR-LINEAR MODEL: JP $=1.8$

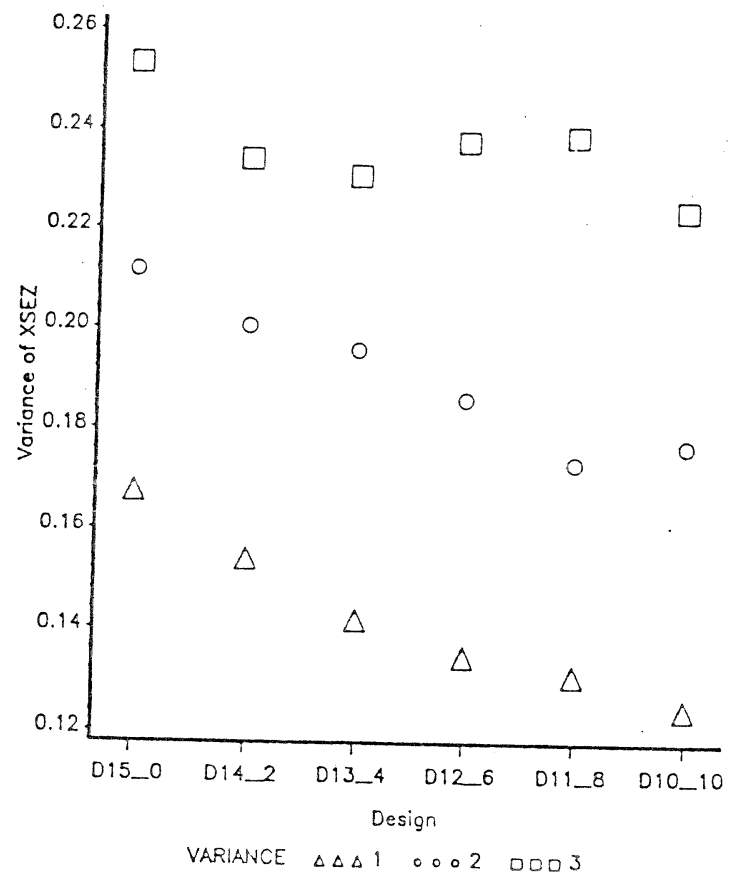

FIGURE 7

RESULTS FROM SIMULATION: 6-POINT DESIGNS 'MAX RESPONSE' OPTIMAL DOSE ESTIMATION TECHNIQUE

LINEAR - LINEAR MODEL: JP $=1.8$

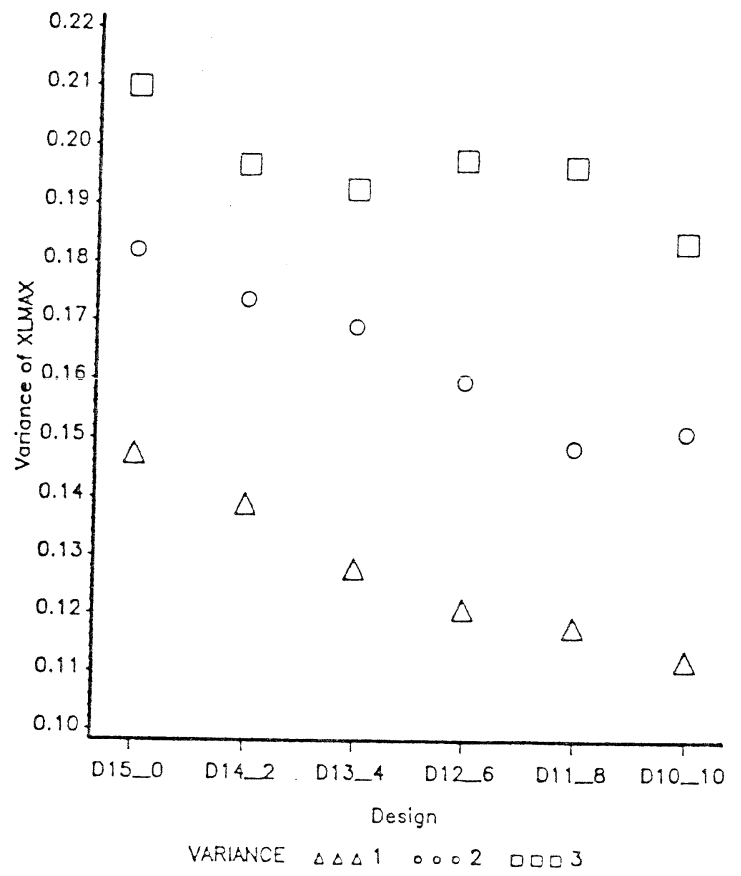

FIGURE B 